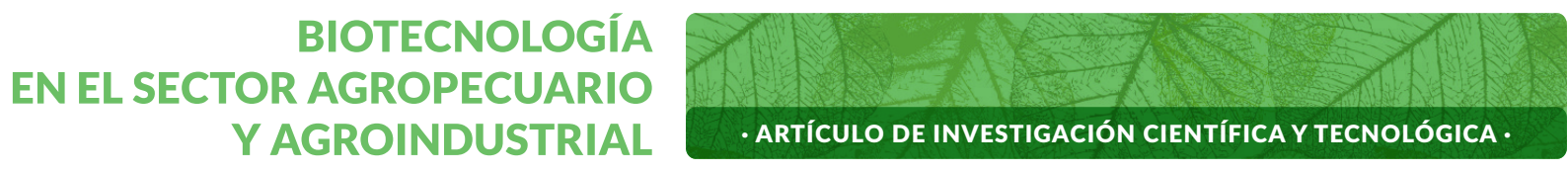

Vol. 18 No 2 · Julio - Diciembre 2020 • ISSN - 1692-3561 · ISSN-e 1909-9959 · DOI: http://dx.doi.org/10.18684

\title{
Elaboración de una bebida a base de suero lácteo y pulpa de Theobroma grandiflorum*
}

\section{Preparation of a beverage based on whey and pulp of Theobroma grandiflorum}

\section{Preparação de uma bebida à base de soro de leite e polpa de Theobroma grandiflorum}

ADRIANA ISABEL RODRÍGUEZ-BASANTES ${ }^{1}$, CRISTIAN AUGUSTO ABAD-BASANTES², AMAURY PÉREZ-MARTÍNEZ3, KAREL DIÉGUEZ-SANTANA ${ }^{*}$

Historial del Artículo

Recibido para evaluación: 30 de Julio 2019.

Aprobado para publicación: 11 de Mayo 2020.

* Proyecto de investigación de orígen: "Elaboración de una bebida a base de suero lácteo con diferentes concentraciones de pulpa de Theobroma grandiflorum", para la obtención al grado de Master en Ingeniería Agroindustrial. Financiación: Mención Sistemas Agroindustriales, en la Universidad Estatal Amazónica: Culminación: el 28 de junio de 2019.

1 Universidad Estatal Amazónica, Facultad de Ciencias de la Tierra. Master en Agroindustrias, Mención Sistemas Agroindustriales. Puyo, Ecuador. ORCID ID: 0000-0002-2532-6504

2 Universidad Estatal Amazónica, Facultad de Ciencias de la Tierra. Master en Ingeniería Agroindustrial. Puyo, Ecuador. ORCID ID: 0000-0003-1250-9974.

3 Universidad Estatal Amazónica, Facultad de Ciencias de la Tierra. Dr. en Ciencias (Ingeniería Química)., Mención Sistemas Agroindustriales. Puyo, Ecuador. ORCID ID: 0000-0003-3978-7982

$4 \quad$ Universidad Estatal Amazónica, Facultad de Ciencias de la Tierra, Master en Ingeniería en Saneamiento Ambiental. Candidato a PhD en Química Industrial, Mención Sistemas Agroindustriales. Puyo, Ecuador. ORCID ID: 0000-0003-4064-0566

Correspondencia: karel.dieguez.santana@gmail.com ,kdieguez@uea.edu.ec

Cómo citar este artículo: ADRIANA ISABEL RODRÍGUEZ-BASANTES, CRISTIAN AUGUSTO ABAD-BASANTES, AMAURY PÉREZ-MARTÍNEZ, KAREL DIÉGUEZ-SANTANA. Clonación del CDNA codificante de las subunidades de la gonadotropina luteinizante de Pimelodus grosskopfii. Revista Biotecnología en el Sector Agropecuario y Agroindustrial,18(2),2020.166-175, DOI:http://dx.doi.org/10.18684/BSAA(18)166-175 


\section{RESUMEN}

La industria láctea genera enormes cantidades de suero de leche que frecuentemente, es vertido como residuo. El presente trabajo tiene como objetivo elaborar una bebida de valor proteico a base de suero de leche con diferentes concentraciones de pulpa, de la fruta amazónica Theobroma grandiflorum (copoazú). Se realizó la caracterización físico-química del suero de leche y se elaboraron tres formulaciones de bebidas variando el porcentaje de pulpa de copoazú (10, 20 y 30\% p/p). Las bebidas lácteas obtenidas se evaluaron en cuanto a $\mathrm{pH}$, la acidez en ácido láctico, grasas, proteínas, recuento de microorganismos y aceptación sensorial. Las tres formulaciones cumplen con los requerimientos físicos, químicos y microbiológicos establecidos en la norma NTE INEN 2609:2012, para bebidas de suero leche y el análisis estadístico no muestra diferencias significativas entre los tratamientos. La evaluación sensorial fue realizada por un panel no entrenado y se consideraron cuatro atributos con base en cincos puntos de la escala hedónica. La formulación de mayor aceptación fue la bebida de $70 \%$ de lactosuero y $30 \%$ de copoazú, y el sabor fue el factor de mayor puntuación. Finalmente, todas las bebidas elaboradas presentan porcentajes proteicos superiores a los requerimientos para bebidas lácteas.

\section{ABSTRACT}

The dairy industry generates huge quantities of whey that is frequently discharged as waste. The present work aims to prepare a protein beverage based on whey with different concentrations of the Amazonian fruit pulp from Theobroma grandiflorum (cupuaçu) The physical-chemical characterization of the whey was carried out and three beverage formulations were prepared by varying the percentage of copoazu pulp $(10,20$, and $30 \% \mathrm{w} / \mathrm{w})$. The milk drinks obtained were evaluated in terms of $\mathrm{pH}$, acidity in lactic acid, fats, proteins, count of microorganisms, and sensory acceptance. The three formulations meet the physical, chemical and microbiological requirements established in the whey beverages standard NTE INEN 2609: 2012, and the statistical analysis does not show significant differences between treatments. The sensory evaluation was carried out by an untrained panel and four attributes were considered based on five points of the hedonic scale. The most widely accepted formulation was the $70 \%$ whey and $30 \%$ cupuaçu drink, and the taste was the highest-scoring factor. Finally, all beverages elaborate have higher protein percentages than the requirements for dairy drinks.

\section{RESUMO}

A indústria de laticínios gera grandes quantidades de soro que é freqüentemente descartado como resíduo. O presente trabalho visa preparar uma bebida à base de proteína à base de soro de leite com diferentes concentrações de polpa, da fruta amazônica Theobroma grandiflorum (cupuaçu). Foi realizada a caracterização físico-química do soro e preparadas três formulações de bebidas, variando a porcentagem de polpa de cupuaçu (10, 20 e 30\% p/p). As bebidas lácteas obtidas foram avaliadas em termos de $\mathrm{pH}$, acidez em ácido

\section{PALABRAS CLAVE:}

Copoazú, Evaluación sensorial, Valor proteico, Lactosuero

\section{KEYWORDS:}

Cupuaçu, Sensory evaluation, Protein value, Whey.

\section{PALAVRAS-CHAVE:}

Cupuaçu, Avaliação sensorial, Valor protéico, Soro de leite. 
lático, gorduras, proteínas, contagem de microrganismos e aceitação sensorial. As três formulações atendem aos requisitos físicos, químicos e microbiológicos estabelecidos na norma NTE INEN 2609: 2012, para bebidas de soro e a análise estatística não mostra diferenças significativas entre os tratamentos. A avaliação sensorial foi realizada por um painel não treinado e quatro atributos foram considerados baseados em cinco pontos da escala hedônica. A formulação mais amplamente aceita foi a bebida com $70 \%$ de soro de leite e $30 \%$ de cupuaçu, $e$ o sabor foi o fator de pontuação mais alto. Finalmente, todas as bebidas fabricadas têm maiores percentagens de proteína do que as exigências para as bebidas lácteas.

\section{INTRODUCCIÓN}

La industria láctea genera alrededor de 200 millones de toneladas de suero de leche por año como consecuencia de la fabricación de queso [1]. Las estadísticas indican que una importante porción de este residuo es descartada como efluente el cual crea un serio problema ambiental debido a que afecta física y químicamente la estructura del suelo, una disminución en el rendimiento de cultivos agrícolas y cuando se desecha en el agua, reduce la vida acuática al agotar el oxígeno disuelto [2].

La eliminación del suero se debe, entre otros aspectos, al desconocimiento de algunos productores sobre las bondades nutricionales de este subproducto y a la dificultad para acceder a las tecnologías apropiadas para su manejo y procesamiento; también a limitaciones en la regulación alimentaria que permitan la apropiada utilización como ingrediente alimenticio. Sin embargo, durante las últimas décadas ha habido un creciente interés en la utilización de suero de leche para la producción de productos de valor añadido, y aprovechar los nutrientes como proteína, lactosa, minerales y vitaminas presentes en el suero de leche [3], por lo que su reutilización es una importante alternativa para la producción de alimentos [4, 5].

Varios autores Cappato et al. (2018) Siqueira, et al. (2013), Amaral, et al. (2018) [6-9], recientemente han propuesto el uso de frutas como un enfoque interesante en la elaboración de bebidas de lactosuero. Por ejemplo Cappato et al. (2018) [6] emplea la pulpa de acerola (Malpighia emarginata DC) en la elaboración de una bebida de suero de leche. Mientras, Amaral et al. (2018) [8, 9] han empleado mezclas de jugos de uvas en preparaciones con $\mathrm{CO}_{2}$ supercrítico y evaluados aspectos sensoriales. Por su parte, Siqueira et al. (2013) [7] argumentan sobre los beneficios del empleo de un $25 \%$ de pulpa de guanábana en formulación de bebidas lácteas, tanto en la preferencia de los consumidores, como en los valores nutritivos, siendo incluso, superiores a bebidas lácteas fermentadas, por lo que la adición de pulpa de fruta, puede incorporar características sensoriales deseables y, además, contribuir a aumentar el valor nutricional.

La fruta copoazú (Theobroma grandiflorum) es una de las nueve especies nativas de la región amazónica del genero Theobroma. La pulpa obtenida de la fruta es de color blanco amarillento, está compuesta de un $61 \%$ de carbohidratos, $6 \%$ de proteína y un $7 \%$ de polisacáridos de pectina [10]. Además, tiene un alto valor nutricional, siendo una fuente de ácido ascórbico (96-111 mg/100 g) y compuestos fenólicos (20,5 mg/100 g) [11] y según Pugliese [12], presenta una mayor actividad anti-oxidante que otras pulpas de fruta que son más comúnmente utilizados para la producción de yogur, tales como pulpa de fresa. Además, es relativamente rica en calcio, fósforo y hierro y presenta un contenido moderado de vitamina C [13].

Faber (2015) y Costa $(2017)[13,14]$ manifiestan que tiene excelentes características tales como aroma, sabor y textura que le confieren un alto potencial económico. Por su sabor distintivo se utiliza como ingrediente para la fabricación de helados, jugos, licores, vinos, jaleas, yogures, mermeladas y otros productos, en lugar de ser consumido en forma natural [15].

El objetivo del presente trabajo fue elaborar una bebida de valor proteico a base de suero de leche con diferentes concentraciones de copoazú, con adecuadas propiedades sensoriales y nutricionales, como una alternativa de ofrecer un valor agregado al lactosuero proveniente de la fabricación de queso. Para ello, se identificaron las principales etapas del proceso de elaboración de la bebida, se caracterizaron los parámetros físicos-químicos y microbiológicos del producto final y finalmente se evaluaron las características nutricionales y sensoriales de la bebida elaborada.

\section{MÉTODO}

Para la elaboración de la bebida se empleó lactosuero descremado sin sal, procedente de la elaboración de 
queso de Industrias de Lácteos "San Salvador" de la Provincia de Chimborazo, Ecuador y pulpa de copoazú (Theobroma grandiflorum), extraída de las frutas de productores locales, de la provincia de Pastaza, Ecuador. Adicionalmente, se empleó azúcar, como edulcorante, $y$ como conservante sorbato de potasio (grado alimentario), mientras como estabilizante se utilizó la gelatina sin sabor (grado alimentario). Todo el proceso de elaboración se realizó en el Laboratorio de Investigación de la Facultad de Ciencias de la Escuela Superior Politécnica de Chimborazo (ESPOCH), ubicado en Av. Panamericana Sur km $11 / 2$ en la ciudad de Riobamba, provincia de Chimborazo, Ecuador, coordenadas, 11'39'36 S, 7840'48' O. Los valores medios de temperatura media y humedad relativa en el mes de la investigación (febrero/2019) fueron de $14,4^{\circ} \mathrm{C}$ y $71,1 \%$, respectivamente.

\section{Elaboración de la bebida a base de suero lácteo con diferentes concentraciones de copoazú}

Formulación del producto. El lactosuero (LS) y la pulpa de copoazú (C) se mezclaron a diferentes proporciones durante la formulación; 90 LS:10 C (F1), 80 LS:20 C (F2) y 70 LS:30 C (F3). El Cuadro 1 muestra los detalles de las cantidades de materias primas necesarias para la elaboración de $1000 \mathrm{~mL}$ de bebida láctea no fermentada. Las cantidades de suero lácteo se establecieron en función de los requerimientos de la norma NTE INEN 2609:2012 (Bebidas de suero leche), mientras que para los conservantes y estabilizantes se consideraron los límites máximos permitidos de la Norma NTE INEN 2074 (Aditivos Alimentarios).

Parámetros establecidos en la Normativa Ecuatoriana. Para la elaboración de la bebida se cumplió con los parámetros regulados en las normas técnicas ecuatorianas. En el caso de la calidad del suero de leche (Materia prima), se siguió la Norma Técnica Ecuatoriana NTE INEN 2594: 2011-08 Suero de leche líquido, tanto para los requisitos fisicoquímicos como microbiológicos. Mientras para la bebida a base de suero de leche

Cuadro 1. Formulaciones de la bebida de lactosuero y copoazú.

\begin{tabular}{|l|l|l|l|}
\hline \multirow{2}{*}{\multicolumn{1}{c|}{ Ingredientes }} & \multicolumn{1}{|c|}{ F1 } & \multicolumn{1}{c|}{ F2 } & \multicolumn{1}{c|}{ F3 } \\
\cline { 2 - 4 } & \multicolumn{3}{c|}{ (gramos) } \\
\hline Lactosuero & 737,5 & 655,5 & 573,8 \\
\hline Pulpa de copoazú & 82,0 & 164,0 & 245,7 \\
\hline Azúcar & 180,0 & 180,0 & 180,0 \\
\hline Sorbato de potasio (conservante) & 0,4 & 0,4 & 0,4 \\
\hline Gelatina (estabilizante) & 0,1 & 0,1 & 0,1 \\
\hline
\end{tabular}

(producto final) se consideraron los requisitos expuestos en la Norma Técnica Ecuatoriana, NTE INEN 2609: 2012-01. Bebida de suero. Requisitos.

\section{Operaciones para el desarrollo de la bebida proteica a base de lactosuero y pulpa de copoazú}

El proceso se inició con una limpieza y desinfección de todos los equipos y materiales. Se seleccionaron las frutas sin daños físicos aparentes, se lavaron y pelaron. Luego, se realizó un despulpado manual con un mazo de madera, para separar la masa del fruto de las semillas. Posteriormente, el lactosuero se filtró con papel filtro Whatman No 4, y se mezcló con la pulpa de copoazú. Después, la mezcla se homogenizó en una licuadora industrial durante 30-60 s. Una vez distribuidos uniformemente todos los ingredientes se procedió a la pasteurización, esta operación se llevó a cabo en una marmita a $75^{\circ} \mathrm{C}$ por un tiempo de 10 min. Inmediatamente, se realizó un proceso de enfriado para causar golpe térmico, se llevó acabo con baño en agua a $6^{\circ} \mathrm{C}$, hasta alcanzar $20^{\circ} \mathrm{C}$. Posteriormente, se adicionó gelatina sin sabor $(0,01 \%)$ (estabilizante) y sorbato de potasio $(0,05 \%)$ (conservante). El producto pasteurizado se envasó manualmente a una temperatura de $20^{\circ} \mathrm{C}$ en botellas de plástico de $250 \mathrm{~mL}$. Finalmente, el producto se almacenó en condiciones normales de refrigeración $\left(4^{\circ} \mathrm{C}\right)$, con la finalidad de mantener las propiedades organolépticas de la bebida.

\section{Evaluación físico-químico y microbiológica del suero y de las bebidas a base de suero de leche y copoazú}

Análisis físico-químico. Los parámetros determinados fueron: $\mathrm{pH}$, acidez, ${ }^{\circ}$ Brix, proteína, y grasa. En el caso del $\mathrm{pH}$, se siguieron los procedimientos descritos en la Norma Técnica (NTE INEN 389) y se empleó un pHMetro Marca Thermo Scientific, modelo Orion Versa Star Pro. La acidez se midió como acidez titulable, expresada como ácido láctico. Se colocaron 20,0 $\mathrm{mL}$ de lactosuero en un Erlenmeyer y se le adicionó $40,0 \mathrm{~mL}$ de agua destilada. Luego se añadieron $2 \mathrm{~cm}^{3}$ de solución indicadora de fenolftaleína y con agitación se tituló con una solución de hidróxido de sodio $(0,1 \mathrm{~N})$, una vez visualizado el color rosado, se continuó añadiendo solución durante 30 segundos. Se tomó lectura del consumo de la solución y se obtuvo el \% de acidez titulable como ácido láctico. Para la determinación de ${ }^{\circ}$ Brix, se pesaron $5 \mathrm{~g}$ de muestra y se diluyó con $50 \mathrm{~mL}$ de agua destilada, se colocó una 
gota de la muestra en el prisma del refractómetro abierto, y se realizó la lectura del porcentaje en peso de azúcar para las soluciones azucaradas o el porciento de sólidos solubles en el caso de tratarse de otras sustancias (puré o pasta de tomate, vinagres, etc.). Por su parte, el contenido de proteína se determinó según los procedimientos descritos en la norma NTE INEN 0016. En el equipo de Kjeldahl, para digestión y destilación se procesaron $5 \mathrm{~g}$ de muestra con ácido sulfúrico concentrado y se realizó la valoración del exceso de ácido, con hidróxido de sodio $(0,1 \mathrm{~N})$, en presencia de rojo de metilo y se empleó el factor de conversión de 6,38. Los principales equipos empleados fueron refractómetro marca Hanna Instruments, Balanza analítica marca Ohaus Pioneer y baño de maría ultrasónico, modelo SONICA. Mientras, los reactivos empleados fueron de grado analítico: Hidróxido de sodio, Sigma aldrich, Grado Reactivo Acs. $\geq 98 \%$, Ácido sulfúrico 98\%, Loba Chemie, Rojo de metilo (Acros Organics) y Fenolftaleina Acs.

Análisis microbiológico. Se realizó el análisis microbiológico de las tres bebidas, aplicando el Método Británico en placas Petrifilm y los resultados fueron comparados con los límites establecidos en la norma (NTE INEN 2609:2012), para bebidas de suero de leche.

En el análisis microbiológico se analizó principalmente la presencia de los siguientes microrganismos: aerobios Mesófilos, Staphylococcus aureus, Escherichia coli. En el caso, de los tres primeros se utilizó el método de recuento en siembra en placas de películas secas rehidratables Mientras, para la E. coli se realizó en un medio de cultivo líquido por el método de determinación del número más probable (NMP) por la técnica de dilución en tubos de acuerdo a las normas NTE INEN 1529-6 y NTE INEN 1529-8.

\section{Evaluación sensorial de las bebidas}

Para conocer el grado de aceptación de la bebida frente a los consumidores se realizó una encuesta organoléptica a un panel no entrenado, compuesto por 30 personas con edades comprendidas entre 18 a 21 años. El modelo empleado, se basó en el color, olor, sabor y textura y estuvo en función de la escala hedónica con base en 5 puntos: Me gusta mucho (5), Me gusta (4), No me gusta ni me disgusta (3), Me disgusta (2), Me disgusta mucho (1). Criterios que también han sido considerados en otras investigaciones $[16,17]$.

\section{Tratamiento de los datos}

Los datos de los diferentes análisis (físico-químico, microbiológico y organoléptico de las pruebas de preferencia y aceptación) fueron analizados estadísticamente utilizando el programa Statistica Versión 8 . Se realizó estadística descriptiva para conocer los valores medios, mínimos, máximos y la desviación estándar. En el caso de distribución normal, se aplicó el análisis de varianza (ANOVA) y la prueba de comparación de tratamientos de Tukey $(p<0,05)$, o la Prueba no paramétrica de Friedman para realizar comparaciones entre las muestras o formulaciones. En los casos que el estadístico Friedman resultó significativo, se analizaron las diferencias entre las variables a través de la prueba de los rangos con signo de Wilcoxon para dos muestras relacionadas.

\section{RESULTADOS}

\section{Evaluación del suero de leche}

Características físico-químicas. El Cuadro 2 muestra los resultados obtenidos de los análisis físico-químicos realizados al suero dulce en la elaboración de las bebidas lácteas. Como se puede apreciar el pH obtenido fue de 6,45 , lo que permite clasificar al lactosuero utilizado como dulce, por provenir de la coagulación enzimática, por uso de enzima coagulante. Mientras el valor de proteína fue de $0,88 \%$, considerándose similar al requerido en la NTE INEN 2594:2011.

Estos resultados son similares al lactosuero empleado en el estudio de Montesdeoca et al. (2017), que reportan valores de $\mathrm{pH}$ de 6,6 , acidez de $0,16 \%$ y proteína de 0,8 [18]. Por su parte, otros estudios como el de Skryplonek et al. (2019), difieren en cuanto al pH

Cuadro 2. Características físico - químicas del suero dulce.

\begin{tabular}{|l|c|c|}
\hline \multicolumn{1}{|c|}{ Parámetros } & Suero dulce & $\begin{array}{c}\text { Valores máximos } \\
\text { permisibles }\end{array}$ \\
\hline Grasa, \% & 0,57 & Hasta 0,3 \\
\hline Temperatura, ${ }^{\circ} \mathrm{C}$ & 20 & \\
\hline $\mathrm{pH}$ & 6,45 & $6,4-6,8$ \\
\hline Acidez, \% & 0,178 & 0,16 \\
\hline Proteína, \% & 0,88 & $>0,8$ \\
\hline 'Brix & 6,2 & \\
\hline
\end{tabular}

*Según NTE INEN 2594: 2011-08 
(valor de 4,85 ) y la acidez $(0,49 \%)$, pero en ese caso es suero ácido [5]. En este estudio los valores de acidez son inferiores y de igual manera el pH es un valor más básico, pues la materia prima es suero dulce.

Evaluación microbiológica. Con el fin de alcanzar la calidad e inocuidad del producto y detectar otros microorganismos que podrían ser causantes de alteraciones microbianas (enfermedades) se efectuaron análisis microbiológicos para revelar la presencia de estos, antes de iniciar con la elaboración de las bebidas lácteas; los resultados se muestran en el Cuadro 3.

Como se puede observar en el Cuadro 3, los parámetros del análisis microbiológico para el suero dulce, se encuentran dentro de los límites establecidos. Por lo que los datos reflejan los resultados positivos de la planta de lácteos "San Salvador", en cuanto a higiene e inocuidad de los alimentos, pudiendo estar asociados con la modernización de su infraestructura, tecnificación de los procesos y la correcta aplicación de normas de higiene que garantizan la inocuidad de los derivados lácteos y los subproductos como el suero.

\section{Características físico-químicas y microbiológicas de las bebidas a base de suero de leche y copoazú}

Como se puede apreciar en el Cuadro 4, las bebidas pasteurizadas F1, F2 y F3, cumplen con los parámetros físico-químicos y microbiológicos que establece la norma NTE INEN 2609:2012, para bebidas de base suero lácteo. En el análisis estadístico de comparación de medias, la formulación 1 muestra diferencias significativas con las formulaciones 2 y 3 en los parámetros proteína láctea y grasa entre el primer tratamiento con el tratamiento 2 y 3 , esto está asociado a que es el tratamiento que contiene mayor cantidad de lactosuero. Sin embargo, en la grasa total no existen diferencias significativas, entre las tres bebidas elaboradas, por lo que la adición de la fruta infiere en el contenido proteico lácteo, pero no en el contenido proteico total de la bebida.

Adicionalmente, todas las formulaciones cumplen con los requerimientos de proteína láctea, según la Normativa Técnica Ecuatoriana que establece un valor mínimo de $0,4 \%$, y en los tres casos evaluados los resultados son superiores a $0,82 \%$, lo que muestra que las bebidas presentan buenos valores proteicos y por tanto pueden ser alimentos adecuados.
Cuadro 3. Resultados del análisis microbiológico del suero dulce pasteurizado.

\begin{tabular}{|l|c|c|}
\hline \multicolumn{1}{|c|}{ Parámetros } & $\begin{array}{c}\text { Suero } \\
\text { dulce }\end{array}$ & $\begin{array}{c}\text { Valores } \\
\text { máximos } \\
\text { permisibles* }\end{array}$ \\
\hline Aerobios mesófilos UFC/mL & 1000 & 30000 \\
\hline E.coli NMP/mL & ausencia & $<10$ \\
\hline Staphylococcus aureus UFC/mL & ausencia & $<100$ \\
\hline
\end{tabular}

* Los valores corresponden al índice máximo permisible para identificar nivel de buena calidad, según NTE INEN 2594: 2011-08.

También, fueron encontradas diferencias en los ${ }^{\circ} \mathrm{Brix}$, entre las formulaciones 1 y 2 con la formulación de $30 \%$ de pulpa de fruta, este último tiene un valor superior que los demás, y esto está asociado a que es la bebida que contiene mayor cantidad de pulpa de fruta y la fruta tiene $61 \%$ de azúcares totales en base seca, por lo que la bebida elaborada contendrá mayor cantidad de azúcares en su composición [10].

Por su parte, el resto de los parámetros, lactosa en el producto parcialmente deslactosado, latosa en el producto bajo en lactosa y acidez no difieren estadísticamente entre las formulaciones elaboradas. Los criterios de porcentajes de lactosa (tanto en el producto parcialmente deslactosado, como en producto bajo en lactosa) de las tres bebidas presentan valores que cumplen con la norma, pues en todos los casos los resultados obtenidos son inferiores a los límites máximos de lactosa de la norma NTE INEN 2609:2012.

Es importante resaltar que las tres bebidas cumplen todos los parámetros físicos-químicos, pero en el caso de la bebida F3 presentan los mayores valores proteicos a pesar de presentar en su composición, menores cantidades de suero lácteo (70\%), por lo que parte de este valor está asociado a la fruta añadida que como plantean [15], posee elevados valores nutricionales (20,6 mg/g en peso seco). Así mismo, la bebida F3 posee los valores menores en cuanto a \% de Lactosa (para producto parcialmente deslactosado, como en producto bajo en lactosa), lo que también puede ser un factor importante para la asimilación de la misma.

Al comparar estos resultados con estudios similares se encuentran diferencias principalmente en la acidez, pues los análisis físico-químicos realizados en otros estudios, como Montesdeoca et al. obtienen una acidez superior $(0,8 \%)$ y Brix de $15 \%$ en las bebidas elaboradas. Por su parte, Thakkar et al. (2018), obtiene 1,71, 
pero en este caso se trata de una bebida fermentada y además presenta un $60 \%$ de jugo de naranja añadido que también incide en el nivel de acidez (1,42\%) [19].

Mientras, Skryplonek et al. (2019) en una bebida fermentada elaborada con $50 \%$ de lactosuero y $50 \%$ de leche y la cepa Bifidobacterium animalis ssp. lactis BB12 , la acidez se mantiene en valores cercanos a 0,5 [5].

Por su parte, los criterios microbiológicos evaluados también muestran resultados positivos, pues en todos los casos y parámetros evaluados (Aerobios mesófilos, Escherichia coli, Staphylococcus aureus, Salmonella/25 g, Detección de Listeria monocytogenes/ 25 g) se encuentran muy por debajo de los límites permisibles. En el caso de los dos últimos, con ausencia de los mismos como rige la norma técnica nacional, para este tipo de bebida.

Los resultados obtenidos están asociados a la calidad de las materias primas empleadas, pues como se mencionó anteriormente, en la evaluación microbiológica el suero de leche cumplía los parámetros. Otro aspecto importante fue que la elaboración se realizó siguiendo las Buenas Prácticas de Manufactura (BPM) y cumpliendo las medidas de higiene e inocuidad necesarias para la elaboración de alimentos.

El análisis microbiológico determina el grado de contaminación al que está expuesto el producto en sus diferentes etapas. Al multiplicarse los microorganismos en el alimento, pueden producir cambios en sus características organolépticas y en su $\mathrm{pH}$, lo que se traduce en alteraciones fáciles de constatar, como rancidez, acidez o alcalinización, putrefacción y aparición de manchas en la superficie. Pero puede ser también que el alimento no presente alteración apreciable, y sin embargo estar contaminado, representando así un riesgo para el consumidor.

\section{Evaluación sensorial}

Los resultados de la evaluación de la bebida, sobre el color, olor, sabor y textura fueron obtenidos siguiendo la escala hedónica con base en cinco (5) puntos. El Cuadro 5 muestra los resultados obtenidos de las diferentes características organolépticas del producto y las diferencias estadísticas.

En el Cuadro 5 se observan los resultados de la calificación para cada atributo, sobresaliendo la formulación 3 (70\% de suero lácteo y $30 \%$ de pulpa de copoazú), pues en todos los atributos presenta los valores superiores (entre 4,63-4,93/5), también presenta los mayores rangos con el color $(2,90)$, olor $(2,88)$, sabor $(2,98)$ y textura $(2,82)$. Al analizar las diferencias de los atributos entre las formulaciones, todos (color, olor, sabor y textura) presentan diferencias significativas (Valores de $p=0,00$ ). También, los coeficientes de concordancia de Kendall son altos, lo que indica que existe concordancia entre los evaluadores, sobresale el atributo sabor que presenta el valor mayor $(0,975)$, muy cercano a la unidad.

Por su parte, en el Cuadro 6 aparecen los valores de la prueba de los rangos con signo de Wilcoxon, que se

Cuadro 4. Resultados de la caracterización físico-química y microbiológica de las bebidas.

\begin{tabular}{|c|c|c|c|c|}
\hline Parámetros/100mL de bebida & F1 & F2 & F3 & Requisitos* \\
\hline Proteína láctea \% & $0,9 \pm 0.01^{a}$ & $0,84 \pm 0.00^{b}$ & $0,82 \pm 0.01^{b}$ & $>0,4$ \\
\hline Grasa, \% & $0,83 \pm 0.00^{\mathrm{a}}$ & $0,77 \pm 0.01^{b}$ & $0,73 \pm 0.01^{b}$ & \\
\hline Proteína \% & $1,59 \pm 0.02^{\mathrm{a}}$ & $1,6 \pm 0.01^{\mathrm{a}}$ & $1,63 \pm 0.01^{\mathrm{a}}$ & \\
\hline Lactosa en el producto parcialmente deslactosado & $1,2 \pm 0.03^{a}$ & $1,2 \pm 0.04^{a}$ & $1.05 \pm 0.03^{a}$ & $<1,4$ \\
\hline Latosa en el producto bajo en lactosa & $0,80 \pm 0.03^{a}$ & $0,70 \pm 0.03^{a}$ & $0,70 \pm 0.03^{a}$ & $<0,85$ \\
\hline${ }^{\circ}$ Brix & $7,25 \pm 0.06^{b}$ & $7,3 \pm 0.06^{b}$ & $7,5 \pm 0.03^{a}$ & \\
\hline Acidez & $0,25 \pm 0.02^{\mathrm{a}}$ & $0,22 \pm 0.01^{\mathrm{a}}$ & $0,23 \pm 0.01^{\mathrm{a}}$ & \\
\hline \multicolumn{5}{|l|}{ Microbiológicos } \\
\hline Aerobios mesófilos UFC/mL & $<10$ & $<10$ & $<10$ & $30000-100000$ \\
\hline Escherichia coli UFC/mL & $<1$ & $<1$ & $<1$ & $<10$ \\
\hline Staphylococcus aureus UFC/mL & $<1$ & $<1$ & $<1$ & $<100$ \\
\hline Salmonella /25g. & - & - & - & - \\
\hline Detección de Listeria monocytogenes/25g & - & - & - & - \\
\hline
\end{tabular}

* Requisitos NTE INEN 2609:2012

${ }^{a b}$ Letras diferentes indican diferencias significativas entre las medias de los tratamientos para la prueba ANOVA, Tukey $(0,05)$ 
Cuadro 5. Estadísticos descriptivos y resultados de la prueba de Friedman para los atributos sensoriales de las tres formulaciones de la bebida de suero lácteo y pulpa de copoazú.

\begin{tabular}{|l|c|c|c|c|}
\hline \multirow{2}{*}{ Código } & \multicolumn{4}{|c|}{ Calificación para cada atributo (Media, \pm DE) } \\
\cline { 2 - 5 } & Color & Olor & Sabor & Textura \\
\hline F1 & $2,83 \pm 0,38$ & $3,03 \pm 0,81$ & $1,23 \pm 0,77$ & $3,1 \pm 1,02$ \\
\hline F2 & $3,4 \pm 0,62$ & $3,7 \pm 0,47$ & $3,53 \pm 0,68$ & $3,4 \pm 0,67$ \\
\hline F3 Prueba de Friedman & $4,73 \pm 0,45$ & $4,63 \pm 0,49$ & $4,93 \pm 0,25$ & $4,77 \pm 0,50$ \\
\hline & \multicolumn{4}{|c|}{ Rango promedio/Suma de los rangos } \\
\hline F1 & $1,23 / 37$ & $1,28 / 38,5$ & $1,03 / 31$ & $1,55 / 46,5$ \\
\hline F2 & $1,87 / 56$ & $1,83 / 55$ & $1,98 / 59,5$ & $1,63 / 49$ \\
\hline F3 & $2,90 / 87$ & $2,88 / 86,5$ & $2,98 / 89,5$ & $2,82 / 84,5$ \\
\hline Chi2 Friedman. & 49,00 & 49,56 & 58,51 & 35,09 \\
\hline Valor de p (5\%) & 0,00 & 0,00 & 0,00 & 0,00 \\
\hline Coeficiente de concordancia de Kendall & 0,817 & 0,826 & 0,975 & 0,585 \\
\hline
\end{tabular}

realizó para conocer las diferencias del análisis sensorial, entre las formulaciones evaluadas, donde existen diferencias significativas en todos los parámetros analizados, excepto en la textura de las formulaciones 1 y 2 ( $p=0,17$ y $z=1,37)$. La información de las pruebas estadísticas no permite excluir ninguno de los factores en los criterios sensoriales, aunque el sabor muestra los mayores valores de $z$, y también en las escalas de valoración, anteriormente abordada.

Este resultado concuerda con otros resultados de análisis organolépticos de bebidas a base de lacto suero. En el caso de Rodríguez et al. (2013), la bebida elaborada con cultivo para yogurt y Lactococcus lactis sub. lactis y pulpa de Maracuyá el panel

evaluador le confirió una excelente calificación [20]. Mientras, en el estudio de Molero-Méndez et al. (2017), las bebidas elaboradas fueron fermentadas y también tuvieron buena aceptación, en todos los parámetros evaluados, y la esencia de coco fue la de mejor valoración [17].

Resultados similares obtuvieron Thakkar et al. (2018) con una bebida probiótica funcional formulada $(60 \%$ Jugo de naranja y $40 \%$ suero lácteo), donde la evaluación sensorial del color, sabor y aceptabilidad general fue superior a 8 de 9 puntos posibles en la valoración de la escala hedónica [19]. Igualmente, Montesdeoca et al. (2017) evaluaron el grado de aceptación de una bebida láctea fermentada utilizando lactosuero como sustituto parcial de leche y los atributos: apariencia, aroma, sabor, textura y calidad general obtuvieron resultados aceptables (superiores a 5 , en una escala de clasificación del 1 al 9) [18].
Cuadro 6. Resultados de la Prueba no paramétrica de los rangos con signo de Wilcoxon para los aspectos sensoriales evaluados de las tres formulaciones $(p<0,05)$

\begin{tabular}{|l|c|c|c|c|c|c|c|c|}
\hline \multirow{2}{*}{ Contraste } & \multicolumn{2}{|c|}{ Color } & \multicolumn{2}{c|}{ Olor } & \multicolumn{2}{c|}{ Sabor } & \multicolumn{2}{c|}{ Textura } \\
\cline { 2 - 8 } & $\mathbf{Z}$ & $\mathbf{p}$ & $\mathbf{Z}$ & $\mathbf{p}$ & $\mathbf{Z}$ & $\mathbf{p}$ & $\mathbf{Z}$ & $\mathbf{p}$ \\
\hline F1-F2 & 3,17 & 0,001 & 3,41 & 0.00 & 4,62 & 0,00 & 1,37 & 0,17 \\
\hline F1-F3 & 4,78 & 0,000 & 4,62 & 0.00 & 4,78 & 0,00 & 4,32 & 0,00 \\
\hline F2-F3 & 4,29 & 0,000 & 4,37 & 0.00 & 4,70 & 0,00 & 4,37 & 0,00 \\
\hline
\end{tabular}

Adicionalmente, como plantean Ramírez-Navas and Castro [21], discriminar entre un atributo $u$ otro, a partir de pruebas sensoriales no es adecuado, pues las sensaciones que puede experimentar un consumidor al ingerir un producto no se producen por un solo sentido, sino que están involucrados distintos estímulos que actúan como respuesta a la estimulación compleja.

\section{CONCLUSIONES}

Se identificaron las etapas del proceso de elaboración de tres formulaciones de bebidas a base de suero de leche, siendo en la etapa de mezclado donde se encuentran las principales diferencias al emplearse concentraciones de copoazú/lactosuero de 10/90\%, $20 / 80 \%$ y $30 / 70 \%$. Las tres formulaciones elaboradas cumplen con todos los los parámetros físicos químicos y microbiológicos establecidos en la norma NTE INEN 2609:2012, para bebidas de suero leche. La formulación de mayor aceptación según los resultados estadísticos de la evaluación sensorial fue la bebida de $70 \%$ de lactosuero y $30 \%$ de copoazú, siendo el sabor, el factor de mayor puntuación de los cuatro evaluados. Todas las bebidas elaboradas pre- 
sentan porcentajes proteicos muy superiores a los requerimientos para bebidas lácteas, por lo que se considera que es posible brindar al consumidor una bebida con importantes características nutricionales.

\section{REFERENCIAS}

[1] SILVA E ALVES, A.T. et al. Probiotic Functional Carbonated Whey Beverages: Development and Quality Evaluation, 4(3), 2018, p. 49.

[2] AHMAD, T. et al. Treatment and utilization of dairy industrial waste: A review. Trends in Food Science and Technology, 882019, p. 361-372. doi: 10.1016/j.tifs.2019.04.003.

[3] GANJU, S. and GOGATE, P.R. A review on approaches for efficient recovery of whey proteins from dairy industry effluents. Journal of Food Engineering, 2152017, p. 84-96. doi: 10.1016/j.jfoodeng.2017.07.021.

[4] ARSIC, S. et al. Economic and ecological profitability of the use of whey in dairy and food industry. Large Animal Review, 24(3), 2018, p. 99-105.

[5] SKRYPLONEK, K., DMYTRÓW, I. and MITUNIEWICZ-MAŁEK, A. Probiotic fermented beverages based on acid whey. Journal of Dairy Science, 102(9), 2019, p. 7773-7780. doi: 10.3168/jds.2019-16385.

[6] CAPPATO, L.P. et al. Whey acerola-flavoured drink submitted ohmic heating processing: Is there an optimal combination of the operational parameters? Food chemistry, 2452018, p. 22-28. doi: 10.1016/j.foodchem.2017.10.019.

[7] SIQUEIRA, A.D.M.O., MACHADO, E.D.C.L. and STAMFORD, T.L.M. Bebidas lácteas com soro de queijo e frutas. Ciência Rural, 43(9), 2013, p. 1693-1700.

[8] AMARAL, G.V. et al. Whey-grape juice drink processed by supercritical carbon dioxide technology: Physicochemical characteristics, bioactive compounds and volatile profile. Food Chemistry, 2392018, p. 697-703. DOI: 10.1016/j. foodchem.2017.07.003.

[9] AMARAL, G.V.et al. Whey-grape juice drink processed by supercritical carbon dioxide technology: Physical properties and sensory acceptance. LWT- Food Science and Technology, 922018, p. 80-86. doi: 10.1016/j.Iwt.2018.02.005.
[10] VRIESMANN, L.C. and. DE OLIVEIRA PETKOWICZ, C.L. Polysaccharides from the pulp of cupuassu (Theobroma grandiflorum): Structural characterization of a pectic fraction, Journal of Carbohydrate and Polymers, 77(1), 2009, p. 72-79. doi: 10.1016/j.carbpol.2008.12.007.

[11] PEREIRA, A.L.F. et al. Impact of fermentation conditions on the quality and sensory properties of a probiotic cupuassu (Theobroma grandiflorum) beverage. Food Research International, 1002017, p. 603-611. doi: 10.1016/j.foodres.2017.07.055.

[12] PUGLIESE, A.G. et al. Flavonoids, proanthocyanidins, vitamin C, and antioxidant activity of Theobroma grandiflorum (Cupuassu) pulp and seeds. Journal of agricultural food chemistry, 61(11), 2013, p. 2720-2728. doi: 10.1021/jf304349u.

[13] COSTA, M.P. et al. Consumer perception, health information, and instrumental parameters of cupuassu (Theobroma grandiflorum) goat milk yogurts. Journal of dairy science, 100(1), 2017, p. 157-168. doi: 10.3168/jds.2016-11315.

[14] FABER, M.A. and OSAKI YUYAMA, L.K. Nectar mix functional based on Amazonian fruits. Journal of Cell Science Therapy, 6(1), 2015, p. 1.

[15] PÉREZ-MORA, W., JORRIN-NOVO, J.V. and MELGAREJO, L.M. Substantial equivalence analysis in fruits from three Theobroma species through chemical composition and protein profiling. Food chemistry, 2402018, p. 496-504. doi: 10.1016/j. foodchem.2017.07.128.

[16] CHAVAN, M. et al. Development of non-dairy fermented probiotic drink based on germinated and ungerminated cereals and legume. LWT Food Science and Technology, 912018, p. 339344. doi: 10.1016/j.Iwt.2018.01.070.

[17] MOLERO-MÉNDEZ, M.S. et al. Sensory evaluation of probiotic fermented beverges based on whey. Revista Cientifica de la Facultad de Ciencias Veterinarias de la Universidad del Zulia, 27(2), 2017, p. 70-77.

[18] MONTESDEOCA, R. et al. Production procedure of a fermented milky drink using lactosuero. Revista Chilena de Nutricion, 44(1), 2017, p. 39-44. doi: 10.4067/S0717-75182017000100006.

[19] THAKKAR, P. et al. Formulation and shelf life study of a whey-based functional beverage containing orange juice and probiotic organisms. International Food Research Journal, 25(4), 2018, p. 1675-1681. 
[20] RODRÍGUEZ, A.P.M., DE PAULA, C.D. and SOTELO, M.M.S. Fermented milk drink from cheese whey with added passion fruit pulp. Revista Tecnica de la Facultad de Ingenieria Universidad del Zulia, 36(3), 2013, p. 202-209.

[21] RAMÍREZ-NAVAS, J.S. and CASTRO, V. Analysis of acceptance and preference of Manjar Blanco del Valle. Biotecnología en el Sector Agropecuario y Agroindustrial, 12(1), 2014, p. 20-27. 